\title{
Metformin use and its effect on gastric cancer in patients with type 2 diabetes: A systematic review of observational studies
}

\author{
PEIWEN LI ${ }^{*}$, CONG ZHANG ${ }^{*}$, PENG GAO, XIAOWAN CHEN, \\ BIN MA, DEHAO YU, YONGXI SONG and ZHENNING WANG \\ Department of Surgical Oncology and General Surgery, \\ The First Hospital of China Medical University, Shenyang, Liaoning 110001, P.R. China
}

Received August 13, 2016; Accepted August 23, 2017

DOI: $10.3892 / \mathrm{ol} .2017 .7370$

\begin{abstract}
Increasing evidence suggests that metformin use is associated with a decreased risk of cancer. The traditional therapies for gastric cancer (GC) are gastrectomy and chemoradiotherapy; however, these therapies may cause certain adverse effects, which affect a patient's quality of life, and the overall survival rate is low. At present, little is known about whether the use of metformin decreases the risk of GC in patients with type 2 diabetes. Therefore, in the present study, a systematic review was performed to analyze the effect of metformin on GC. A literature search was conducted in PubMed, EMBASE, and the Cochrane Library databases for articles published up to June 30th, 2016. The studies that evaluated GC patients treated with metformin and compared them with GC patients treated with other antidiabetic drugs were reviewed. Eligible studies were evaluated using the Newcastle-Ottawa Scale. Adjusted hazard ratio and $95 \%$ confidence intervals were determined to evaluate the effect of metformin on GC. From the 422 articles evaluated, 5 studies involving a total of 1,804,479 patients met the inclusion criteria and were qualitatively analyzed. The quality of all selected articles was classified as moderate. These studies reported that the long-term use of metformin was associated with a lower risk of GC compared with the lack of use of metformin or the use of other hypoglycemic drugs. In GC patients with diabetes who were subjected to gastrectomy, the cumulative use of metformin reduced the rates of disease recurrence and of all-cause and cancer-specific mortality. Despite the limited number of studies on this subject, currently available evidence indicates that metformin is associated with a decreased risk of GC and improves survival in patients
\end{abstract}

Correspondence to: Professor Zhenning Wang, Department of Surgical Oncology and General Surgery, The First Hospital of China Medical University, 155 North Nanjing Street, Shenyang, Liaoning 110001, P.R. China

E-mail: josieon826@sina.cn

${ }^{*}$ Contributed equally

Key words: metformin, gastric cancer, diabetes, systematic review with type 2 diabetes. However, more well-designed trials are required to elucidate this association.

\section{Introduction}

Metformin is a first-line oral hypoglycemic drug and the most widely used drug for the treatment of type 2 diabetes (1). Metformin is easily accessed in clinical practice owing to its low-cost (2) and mild adverse drug reactions (3). The use of metformin has also been reported to reduce the risk of certain types of cancer in patients with type 2 diabetes mellitus (DM) (4-7). Preclinical studies have indicated that metformin can inhibit the proliferation of cancer cells in vitro and in vivo (8), and simultaneously induce apoptosis (9). Numerous clinical studies have reported that metformin has an antineoplastic effect on several types of malignancy, including colon cancer (4), gastric cancer (GC) (10), pancreatic cancer (11), breast cancer (12), and prostate cancer (5).

$\mathrm{GC}$ is one of the most common cancer types and the second-leading cause of cancer-associated morbidity (13) owing to its high degree of malignancy, and the recurrence and mortality rates are high, even following radical resection and adjuvant radiation and chemotherapy. The 5-year overall survival (OS) rate is $<25 \%$ (14).

To the best of our knowledge, no comprehensive systematic review has reported the effect of metformin use on GC patients with diabetes. Therefore, in the present study, a systematic review was conducted to elucidate the potential effect of metformin on GC among diabetic patients.

\section{Materials and methods}

Search strategy. Relevant articles were searched in major medical databases, including PubMed (www.ncbi.nlm.nih. gov/pubmed; date of access, June 30 2016), EMBASE (www. embase.com; date of access, June 30 2016) and the Cochrane Library (www.cochranelibrary.com; date of access, June 30 2016). All English-language articles published prior to June 30th 2016 were searched using the keywords 'metformin', 'dimethylbiguanide', 'metformin hydrochloride', 'metformin $\mathrm{HCl}$ ', 'gastrointestinal neoplasms', 'digestive system neoplasms', 'stomach neoplasms', 'gastric cancer', and 'gastric neoplasms'. The references of the relevant meta-analyses 
and systematic reviews were then examined to identify other potentially relevant studies.

Inclusion and exclusion criteria. The following studies met the inclusion criteria: i) Studies that evaluated GC patients treated with metformin and compared them with patients who used other antidiabetic drugs; ii) studies that evaluated the association between metformin and $\mathrm{GC}$ using the hazard ratio (HR) or adjusted HR and 95\% confidence intervals (CIs); and iii) retrospective or prospective cohort studies, randomized clinical trials, and case-control studies. If the data were from the same authors or institutes, only the most recent article or the highest-quality article was included. The following types of articles were excluded: i) Reviews, letters, conference abstracts, and comments; ii) preclinical experiments on metformin and GC in vitro and in vivo; and iii) studies that evaluated the use of metformin for other metabolic or endocrine diseases, such as endometriosis and polycystic ovary syndrome.

Data extraction and quality assessment. Two authors (Drs Peiwen Li and Cong Zhang) independently reviewed the included articles and extracted data on the study authors, year of publication, country, study design, number of participants, patient age, follow-up, control groups (treatment with insulin, sulfonylurea or thiazolidinedione, among others), adjusting variables, adjusted hazard ratio (AHR) and 95\% CI, and conclusions (Table I). Any disagreement between the two authors was resolved by consulting a third author. The Newcastle-Ottawa Scale (NOS) (15) was used to assess the quality of the studies. NOS scores $\geq 7$ (NOS scores $=7,8,9)$ were defined as high quality studies. NOS scores $\geq 4$ were defined as moderate quality studies (NOS scores $=4,5,6)$ and NOS scores $=1,2,3$ were defined as low quality studies (Table II).

Statistical analysis. Statistical analysis was performed with Stata software (version 12.0; StataCorp, College Station, TX, USA). The AHR and 95\% CI were used to estimate the association between metformin and $\mathrm{GC}$ risk, and $\mathrm{P}<0.05$ was considered to indicate statistical significance. A P value $<0.01$ with an $\mathrm{I}^{2}$ value of $>50 \%$ indicated substantial heterogeneity. The inverse variance method with a fixed-effects model was applied if no heterogeneity was detected, whereas a random-effects model was used in the alternative cases.

\section{Results}

Search results. A total of 422 studies were initially identified, and 98 were excluded owing to duplication. After reviewing titles and abstracts, an additional 306 articles were excluded. The full texts of 18 studies were then thoroughly reviewed. Of these, 13 articles were removed due to the publication type. Therefore, 5 studies were eventually included in the analysis. No additional articles from the references were added to this review. Two randomized controlled trials (RCTs) [A Diabetes Outcome Progression Trial (ADOPT) (16) and Rosiglitazone Evaluated for Cardiovascular Outcomes and Regulation of Glycemia in Diabetes (RECORD) (17)] were excluded from this review: In ADOPT, only one GC patient was included, and no additional detailed information was available to be extracted for comparison; and in RECORD, the gastric and intestinal malignancies were counted together, which prevented the determination of the number of cases of GC. The flow chart of the selection criteria is shown in Fig. 1.

Study characteristics. All the selected articles were cohort studies, including four retrospective cohort studies and one prospective cohort study. The populations of the studies came from European countries $(18,19)$ and Asian countries $(10,20,21)$. Two included studies focused on GC, whereas the other three studies evaluated other general cancer types in addition to GC. The heterogeneity among the articles was extensive, and included differences in the definition of the subgroups, follow-up period, drug comparators, dosage, and duration of treatment.

Metformin and risk of $G C$. Four studies were based on data from national or local health databases, or health insurance population-based databases, and therefore the bias of selection was almost negligible. However, these studies did not evaluate the same confounding factors, including body mass index (BMI), obesity, glucose level, glycated hemoglobin (HbA1c), smoking history, lifestyle, dietary habits, and Helicobacter pylori infection, as the databases containing all the information necessary to estimate the effect of metformin were unavailable.

Valent (18) compared the effect of the use of metformin together with other oral hypoglycemic drugs (sulfonylureas, $\alpha$-glucosidase inhibitors, and thiazolidinediones, not including insulin) on the risk of various digestive cancers. These authors found that type 1 or type 2 diabetes was associated with increased risk of digestive cancer. Metformin use reduced the risk of the majority of digestive cancers, including GC (AHR, $0.990 ; 95 \%$ CI, 0.986-0.994). Of note was that the effect of sulfonylurea on GC was similar to that of metformin (AHR, 0.989; 95\% CI, 0.981-0.997).

Kim et al (10) distinguished metformin users from regular users of insulin and non-users of insulin. In the non-users of insulin cohort, patients treated with metformin had a significantly lower rate of GC compared with those not treated with metformin $(\mathrm{P}=0.047)$; whereas no significant difference in the rate of GC was observed among the regular users of insulin $(\mathrm{P}=0.379)$. In the group of non-users of insulin, the authors divided the duration of metformin use into four time intervals and detected a $43 \%$ decrease in the risk of GC in DM patients who had used metformin for $>3$ years, after adjusting for covariates (AHR, 0.57; 95\% CI, 0.37-0.87; P=0.009).

Ruiter et al (19) compared the risk of general cancer between patients using metformin and those using sulfonylurea derivatives, and found that the long-term use of metformin was associated with a lower probability of cancer in general compared with the use of sulfonylurea derivatives. Metformin presented a lower risk of GC compared with sulfonylurea derivatives (AHR, 0.83; 95\% CI, 0.76-0.90). The authors emphasized that the lower risk of cancer in the metformin group and the actual decrease in the risk of cancer by metformin were two different concepts. The comparison of models on the basis of different covariables indicated that the risk of cancer was lower in the metformin group compared with the sulfonylurea group; however, this result did not prove that metformin decreased the risk of cancer. 







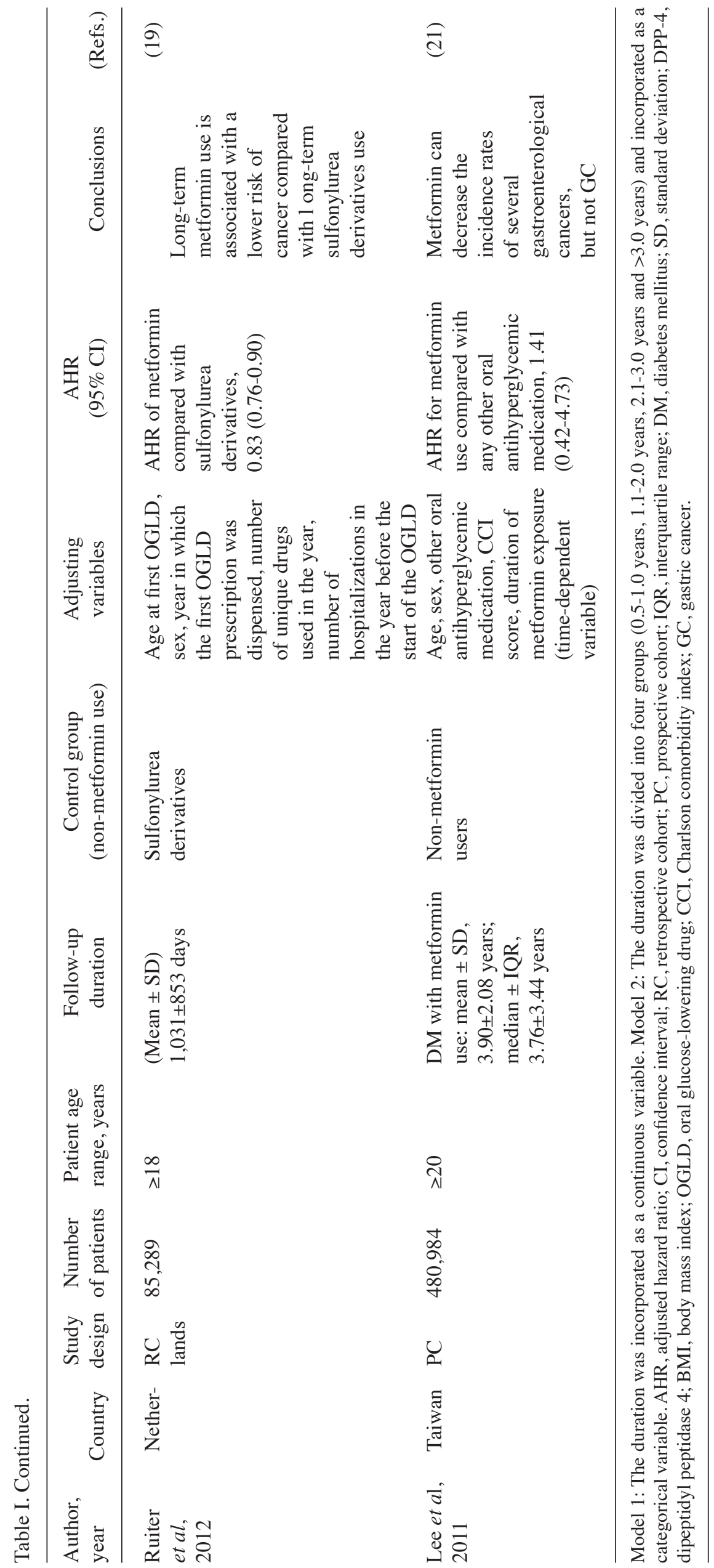


Table II. Assessment of the risk of bias in each cohort study using the Newcastle-Ottawa scale.

\begin{tabular}{|c|c|c|c|c|c|c|c|c|c|c|c|c|}
\hline \multirow[b]{2}{*}{ Author, year } & \multicolumn{4}{|c|}{ Selection } & \multicolumn{2}{|c|}{ Comparability } & \multicolumn{3}{|c|}{ Outcome } & \multirow[b]{2}{*}{ Total } & \multirow[b]{2}{*}{ Quality } & \multirow[b]{2}{*}{ (Refs.) } \\
\hline & REC & SNEC & $\mathrm{AE}$ & DO & $\mathrm{SC}$ & $\mathrm{AF}$ & $\mathrm{AO}$ & $\mathrm{FU}$ & AFU & & & \\
\hline Lee et $a l, 2016$ & - & + & + & + & - & - & + & + & - & 5 & Moderate & (20) \\
\hline Valent, 2015 & + & + & + & + & - & - & + & + & - & 6 & Moderate & (18) \\
\hline Kim et al, 2014 & + & + & + & + & - & - & + & + & - & 6 & Moderate & (10) \\
\hline Ruiter et al, 2012 & + & + & + & + & - & - & + & + & - & 6 & Moderate & (19) \\
\hline Lee et $a l, 2011$ & + & + & + & + & - & - & + & + & - & 6 & Moderate & (21) \\
\hline
\end{tabular}

REC, representativeness of the exposed cohort; SNEC, selection of the non-exposed cohort; AE, ascertainment of exposure; DO, demonstration that outcome of interest was not present at start of study; SC, study controls for age and sex; AF, study controls for any additional factors; AO, assessment of outcome; FU, follow-up of sufficient duration; AFU, adequacy of follow-up of cohorts.

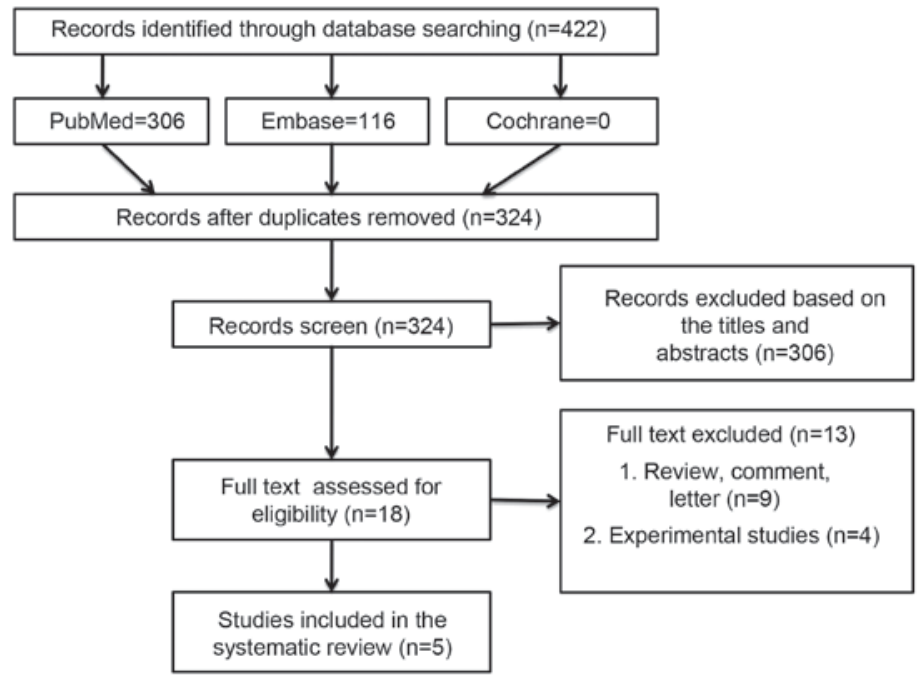

Figure 1. Flow chart of study selection.

Lee et al (21) performed a prospective cohort study in Taiwan and found that metformin treatment reduced the incidence of total and specific cancers (colorectal, liver, and pancreatic) compared with the absence of treatment with metformin. For GC, their results disagreed with those of previous studies, and metformin did not significantly reduce the incidence of cancer (AHR, 1.41; 95\% CI, 0.42-4.73).

In the present study, the overall effect of metformin on the risk of $\mathrm{GC}$ was determined by conducting a meta-analysis using HRs combined with $95 \%$ CIs. The risk of GC in the groups that used metformin cumulatively was lower than that in other study groups, although there was no statistically significant difference (overall HR, 0.867; 95\% CI, 0.726-1.035; $\mathrm{I}^{2}=87.2 \%$; Fig. 2).

Metformin and survival of GC. Lee et al (20) evaluated 1,974 patients with GC who were subjected to curative gastrectomy, and found that the non-diabetic patients had markedly better OS, cancer-specific survival (CSS), and recurrence-free survival (RFS) rates compared with the diabetic patients. By contrast, the prognosis of patients with DM treated with metformin was significantly more favorable than in those not treated with metformin [OS HR, 0.584 (95\% CI, 0.369-0.926); CSS HR, 0.57 (95\% CI, 0.334-0.975); RFS HR, 0.633 (95\% CI, 0.410-0.977)]. The improvement in the survival of DM patients treated with metformin was similar to that of non-diabetic patients, particularly in stage III patients. Furthermore, the results of the multivariable analysis indicated that the decreased risks of recurrence, cancer-specific mortality, and all-cause mortality were strongly associated with each additional 6 months of metformin use [OS AHR, $0.870(95 \% \mathrm{CI}$, 0.801-0.845); CSS AHR, 0.865 (95\% CI, 0.782-0.958); RFS AHR, 0.864 (95\% CI, 0.797-0.937)]. As there was only one study regarding the effect of metformin on the survival of GC, a meta-analysis on this was not conducted.

\section{Discussion}

Several epidemiological studies have observed an increased risk of cancer (22-26), including stomach cancer (27-29), colon, breast, endometrial, liver, pancreatic and bladder cancer $(30,31)$, and prostate cancer (32), in DM patients; however, the precise underlying mechanisms have not been elucidated. Factors such as insulin resistance, secondary 


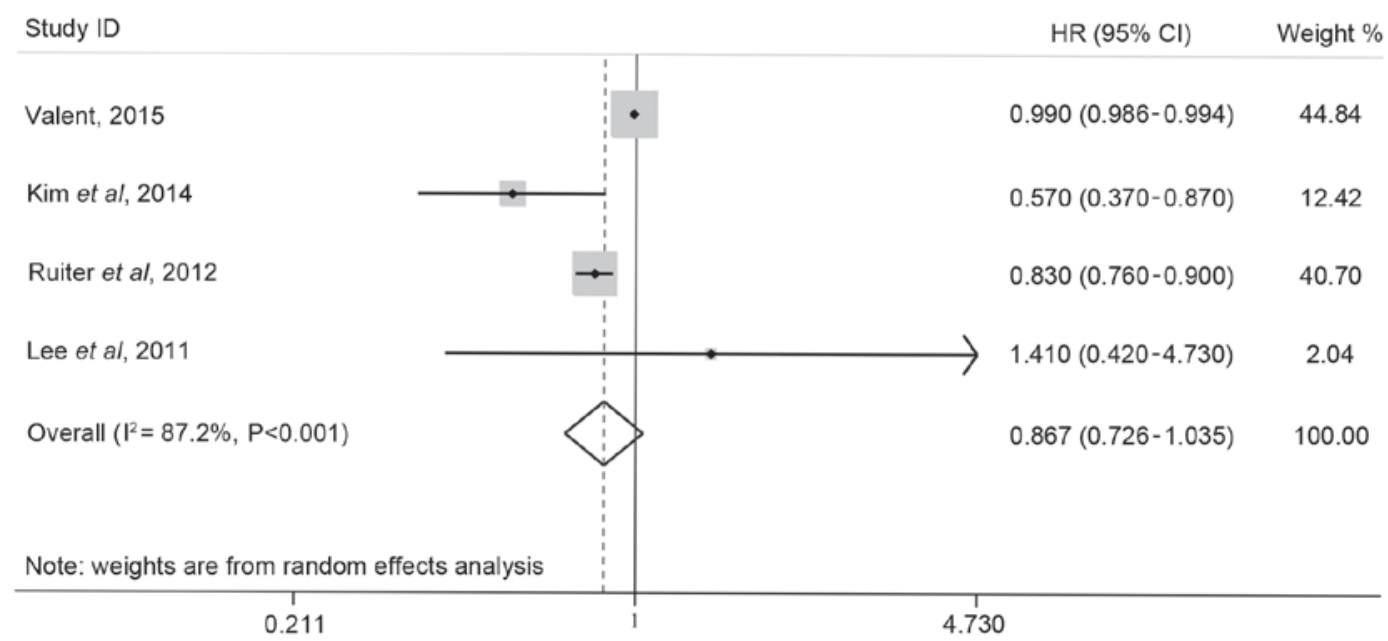

Figure 2. Forest plots of metformin use and gastric cancer risk.

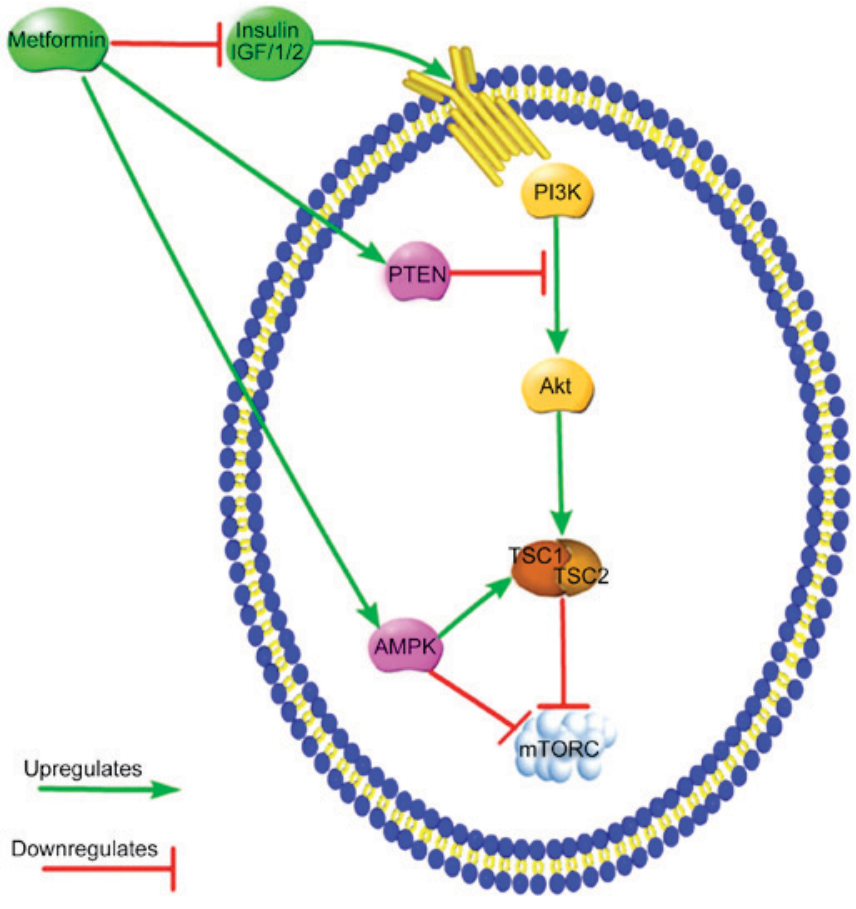

Figure 3. Potential mechanism of the effect of metformin on cancer cells.

hyperinsulinemia, sex hormones and inflammatory cytokines may be associated with each other and may promote tumor cell proliferation in vitro and in vivo via insulin or insulin-like growth factor (IGF) pathways (26,33). A few studies reported that diabetes or hyperglycemia was a tumor-promoting factor for GC. Ikeda and Kiyohara (34) showed that elevated fasting blood glucose greatly increased the incidence of GC and, although other risk factors were adjusted for, including $H$. pylori infection, a moderate increase in the HbA1c level remained a promoter of GC. Zhou et al (35) determined that hyperglycemia promoted the progression of $\mathrm{GC}$ via aquaporin 3 (AQP3); the in vitro expression of AQP3 increased when the glucose levels increased, and AQP3 was overexpressed in GC, leading to the increased migration of cancer cells.

Metformin is the most widely prescribed oral antidiabetic drug for patients with type 2 diabetes. Increasing evidence has demonstrated the anticancer capacity of metformin. A recent meta-analysis by Wu et al (36) in 2015 evaluated the use of metformin in patients with type 2 diabetes and indicated that this use was associated with 14 and $30 \%$ reductions in the rates of cancer incidence and mortality, respectively. Furthermore, other meta-analyses (37-40) obtained similar results, suggesting an overall decrease in the risk of cancer by metformin.

Although several laboratory and epidemiological studies have shown that metformin may serve a general antitumor role in DM patients, it remains unclear whether metformin can reduce the risk of specific types of cancer, such as GC. Therefore, this systematic review was conducted to elucidate this issue. Three studies $(10,18,19)$ found that metformin reduced the risk of GC, whereas one study (21) showed that metformin could reduce the risk of other cancers, excluding GC. A recent clinical study (20) reported that metformin improved the survival of GC in diabetic patients subjected to gastrectomy by decreasing the recurrence, all-cause mortality, and cancer-specific mortality rates.

Mammalian target of rapamycin (mTOR) is a downstream target and intersection of numerous signaling pathways and functions as a modulator of protein synthesis, cellular growth, cellular proliferation, autophagy and metabolism. mTOR is composed of mTOR complex (mTORC) 1 and mTORC2. Insulin or IGF1/2 can activate the phosphatidylinositide 3-kinase (PI3K)-Akt-mTOR signaling pathway primarily via $\mathrm{mTORC1}$, thus promoting protein synthesis and cellular growth and proliferation $(41,42)$. Metformin may indirectly reduce the circulating insulin levels by increasing the sensitivity of peripheral tissues to insulin, thereby impairing the insulin or IGF pathways (42). Phosphatase and tensin homolog deleted on chromosome ten (PTEN) functions as a cancer suppressor by negatively regulating Akt via interruption of the upstream signal from PI3K, and metformin may exert its effect by increasing the level of PTEN and inhibiting mTORC1 expression (43). Metformin directly activates adenosine monophosphate-activated protein kinase (AMPK), which induces the suppression of mTORC1 expression, ultimately decreasing cell growth and proliferation (44). Tuberous sclerosis complex (TSC)1 and TSC2 are proteins encoded by the mutated tumor-suppressor gene in TSC disease (45); 
the TSC1/2 complex inhibits mTORC1 activity, while Akt suppresses the activity of TSC2 by phosphorylation (46). AMPK promotes the formation of the TSC1/2 complex, which inhibits mTORC1 activity (47). Therefore, PTEN and AMPK can suppress tumors by targeting upstream molecules of the mTOR pathway (Fig. 3).

Zhou et al (48) conducted a review and found that metformin could regulate miRNA directly and subsequently modulate downstream genes to prevent oncogenesis and therapy. Kato et al (8) found that metformin could inhibit GC cell proliferation and tumor growth by depressing cell-cycle factors via the modulation of miRNA expression in vitro and in vivo. In addition, these authors reported that different treatment periods and metformin concentrations may lead to differential miRNA expression, which suggests that the duration and dose of metformin should be considered on an individual basis. Yu et al (43) showed that metformin inhibited cell proliferation and tumor growth by targeting certain essential genes that affect tumor growth, proliferation and metastasis. The authors suggested that the dosage of metformin should be kept low, and that treatment should be continuous. The results of the studies by Kato et al (8) and $\mathrm{Yu}$ et al (43) agreed with those of Lee et al (20) and Kim et al (10), who proposed that the long-term use of metformin could improve survival and significantly reduce the risk of $\mathrm{GC}$.

Two of the studies $(18,19)$ included in this review evaluated another type of common hypoglycemic drug, sulfonylureas or sulfonylurea derivatives. Sulfonylureas are frequently prescribed with metformin for type 2 DM. As insulin secretagogues, sulfonylureas increase insulin and IGF levels, which promote tumorigenesis (49); however, it is still debatable whether sulfonylurea derivatives can increase the risk of cancer (50). Glibenclamide was the first second-generation sulfonylurea drug, and it functions by targeting sulfonylurea receptors (SURs). To date, a number of studies have indicated that glibenclamide can inhibit the growth of various types of cancer (51-54); however, the antitumor mechanisms have not been fully elucidated. In recent years, certain studies have focused on potassium $\left(\mathrm{K}^{+}\right)$channels, which are involved in physiological cellular functions, including insulin release, cell proliferation, and apoptosis. Therefore, the regulation of $\mathrm{K}^{+}$channels may form the basis of a therapeutic method for cancer (55-57). Adenosine triphosphate-sensitive potassium channels $\left(\mathrm{K}_{\mathrm{ATP}}\right.$ channels) in the plasma and mitochondrial membrane are made of SURs, and glibenclamide may target SURs by closing $\mathrm{K}_{\mathrm{ATP}}$ channels, leading to cell damage and apoptosis (58).

A cohort study by Yang et al (59) involving 6,103 Chinese patients in Hong Kong showed that the use of gliclazide and glibenclamide may reduce the risk of cancer in a dose-dependent manner. Additionally, a meta-analysis by Thakkar et al (37) demonstrated that metformin use reduced cancer risk (supported by cohort and case-control studies but not by RCTs) and that sulfonylurea use was associated with an increase in all-cancer risk (supported by cohort studies, although case-control studies and RCTs did not prove this association). Ruiter et al (19) hypothesized that it is less likely that sulfonylureas are a cancer-promoting factor. These authors found that the outcomes of patients treated with a combination of metformin and sulfonylureas were similar to those of patients treated with metformin alone. Notably, Valent (18) found that sulfonylureas had a unique protective association with the stomach and pancreas, and that sulfonylureas did not increase the incidence of any type of cancer.

In contrast to the results of other studies, Lee et al (21) found that metformin had no correlation with a reduced risk of GC. The possible reasons for this are as follows: i) The duration of metformin varied significantly among the studies, which prevented the accurate evaluation of the cumulative effect [duration of treatment with metformin, 931 \pm 765 days (mean \pm standard deviation); 755 $\pm 1,200$ days (median \pm interquartile range)]; and ii) certain underlying complementary drugs, such as aspirin (60), vitamin D (61), and statins (62), which have been found to be associated with cancer risk, may have confounded the effect of metformin.

There were a number of limitations to the current study. No RCTs have reported the specific risk of GC in patients treated with metformin; therefore, the analyses were based on retrospective and prospective cohort studies, and strong conclusions could not be made because of the intrinsic heterogeneity of these analyses. Only five articles met the inclusion criteria of this study. The data of the four studies $(10,18,19,21)$ included herein were obtained from national or local health databases or health insurance companies, from which critical data were not available, causing heterogeneity in the results.

To the best of our knowledge, this is the first systematic review to evaluate the effect of metformin on the risk of GC. Although only a few studies have focused on this subject to date, the current evidence indicates that metformin is associated with reduced risk of GC and improved survival in patients with type 2 diabetes. However, more well-designed trials are required to elucidate the anticancer effect of metformin. Metformin does not decrease normal blood sugar level and, for this reason, it may be used by non-diabetic individuals as a protective agent against cancer. However, large, rigorously designed, and high-quality clinical trials are necessary to validate this possible effect.

\section{Acknowledgements}

This study was funded by the Natural Science Foundation of Liaoning Province (grant no. 2014029201), the Program of Education Department of Liaoning Province (grant no. L2014307), the Key Laboratory Programme of Education Department of Liaoning Province (grant no. LZ2015076) and the Scientific Programme of Science \& Technology Department of Liaoning Province (grant no. 2015225002).

\section{References}

1. Nathan DM, Buse JB, Davidson MB, Ferrannini E, Holman RR, Sherwin R and Zinman B; American DiabetesAssociation; European Association for the Study of Diabetes: Medical management of hyperglycaemia in type 2 diabetes mellitus: A consensus algorithm for the initiation and adjustment of therapy: A consensus statement from the American Diabetes Association and the European Association for the Study of Diabetes. Diabetologia 52: 17-30, 2009.

2. Decensi A, Puntoni M, Goodwin P, Cazzaniga M, Gennari A, Bonanni B and Gandini S: Metformin and cancer risk in diabetic patients: A systematic review and meta-analysis. Cancer Prev Res (Phila) 3: 1451-1461, 2010. 
3. Goodwin PJ, Ligibel JA and Stambolic V: Metformin in breast cancer: Time for action. J Clin Oncol 27: 3271-3273, 2009.

4. Sehdev A, Shih YC, Vekhter B, Bissonnette MB, Olopade OI and Polite BN: Metformin for primary colorectal cancer prevention in patients with diabetes: A case-control study in a US population. Cancer 121: 1071-1078, 2015.

5. Tseng $\mathrm{CH}$ : Metformin significantly reduces incident prostate cancer risk in Taiwanese men with type 2 diabetes mellitus. Eur J Cancer 50: 2831-2837, 2014.

6. El-Benhawy SA and El-Sheredy HG: Metformin and survival in diabetic patients with breast cancer. J Egypt Public Health Assoc 89: 148-153, 2014.

7. Tseng $\mathrm{CH}$ : Metformin may reduce oral cancer risk in patients with type 2 diabetes. Oncotarget 7: 2000-2008, 2016.

8. Kato K, Gong J, Iwama H, Kitanaka A, Tani J, Miyoshi H, Nomura K, Mimura S, Kobayashi M, Aritomo Y, et al: The antidiabetic drug metformin inhibits gastric cancer cell proliferation in vitro and in vivo. Mol Cancer Ther 11: 549-560, 2012.

9. Han G, Gong H, Wang Y, Guo S and Liu K: AMPK/mTOR-mediated inhibition of survivin partly contributes to metformin-induced apoptosis in human gastric cancer cell. Cancer Biol Ther 16 : 77-87, 2015.

10. Kim YI, Kim SY, Cho SJ, Park JH, Choi IJ, Lee YJ, Lee EK, Kook MC, Kim CG, Ryu KW and Kim YW: Long-term metformin use reduces gastric cancer risk in type 2 diabetics without insulin treatment: A nationwide cohort study. Aliment Pharmacol Ther 39: 854-863, 2014.

11. Sadeghi N, Abbruzzese JL, Yeung SC, Hassan M and Li D Metformin use is associated with better survival of diabetic patients with pancreatic cancer. Clin Cancer Res 18: 2905-2912, 2012.

12. Xu H, Chen K, Jia X, Tian Y, Dai Y, Li D, Xie J, Tao M and Mao Y: Metformin use is associated with better survival of breast cancer patients with diabetes: A meta-analysis. Oncol 20: 1236-1244, 2015.

13. Yamamoto $\mathrm{H}$, Watanabe $\mathrm{Y}$, Maehata $\mathrm{T}$, Morita R, Yoshida $\mathrm{Y}$, Oikawa R, Ishigooka S, Ozawa S, Matsuo Y, Hosoya K, et al: An updated review of gastric cancer in the next-generation sequencing era: Insights from bench to bedside and vice versa. World J Gastroenterol 20: 3927-3937, 2014.

14. Durães C, Almeida GM, Seruca R, Oliveira C and Carneiro F: Biomarkers for gastric cancer: Prognostic, predictive or targets of therapy? Virchows Arch 464: 367-378, 2014.

15. Stang A: Critical evaluation of the Newcastle-Ottawa scale for the assessment of the quality of nonrandomized studies in meta-analyses. Eur J Epidemiol 25: 603-605, 2010.

16. Kahn SE, Haffner SM, Viberti G, Herman WH, Lachin JM, Kravitz BG, Yu D, Paul G, Holman RR and Zinman B Diabetes Outcome Progression Trial (ADOPT) Study Group: Rosiglitazone decreases C-reactive protein to a greater extent relative to glyburide and metformin over 4 years despite greater weight gain: Observations from a Diabetes Outcome Progression Trial (ADOPT). Diabetes Care 33: 177-183, 2010.

17. Home PD, Kahn SE, Jones NP, Noronha D, Beck-Nielsen H and Viberti G; ADOPT Study Group; RECORD Steering Committee.: Experience of malignancies with oral glucose-lowering drugs in the randomised controlled ADOPT (A Diabetes Outcome Progression Trial) and RECORD (Rosiglitazone Evaluated for Cardiovascular Outcomes and Regulation of Glycaemia in Diabetes) clinical trials. Diabetologia 53: 1838-1845, 2010.

18. Valent F: Diabetes mellitus and cancer of the digestive organs: An Italian population-based cohort study. J Diabetes Complications 29: 1056-1061, 2015.

19. Ruiter R, Visser LE, van Herk-Sukel MP, Coebergh JW, Haak HR, Geelhoed-Duijvestijn PH, Straus SM, Herings RM and Stricker BH: Lower risk of cancer in patients on metformin in comparison with those on sulfonylurea derivatives: Results from a large population-based follow-up study. Diabetes Care 35 119-124, 2012

20. Lee CK, Jung M, Jung I, Heo SJ, Jeong YH, An JY, Kim HI, Cheong JH, Hyung WJ, Noh SH, et al: Cumulative metformin use and its impact on survival in gastric cancer patients after gastrectomy. Ann Surg 263: 96-102, 2016.

21. Lee MS, Hsu CC, Wahlqvist ML, Tsai HN, Chang YH and Huang YC: Type 2 diabetes increases and metformin reduces total, colorectal, liver and pancreatic cancer incidences in Taiwanese: A representative population prospective cohort study of 800,000 individuals. BMC cancer 11: 20, 2011.
22. Lo SF, Chang SN, Muo CH, Chen SY, Liao FY, Dee SW, Chen PC and Sung FC: Modest increase in risk of specific types of cancer types in type 2 diabetes mellitus patients. Int J Cancer 132: $182-188,2013$.

23. Zhang PH, Chen ZW, Lv D, Xu YY, Gu WL, Zhang XH, Le YL, Zhu $\mathrm{HH}$ and Zhu YM: Increased risk of cancer in patients with type 2 diabetes mellitus: A retrospective cohort study in China. BMC Public Health 12: 567, 2012.

24. Yeh HC, Platz EA, Wang NY, Visvanathan K, Helzlsouer KJ and Brancati FL: A prospective study of the associations between treated diabetes and cancer outcomes. Diabetes Care 35: 113-118, 2012.

25. Lee MY, Lin KD, Hsiao PJ and Shin SJ: The association of diabetes mellitus with liver, colon, lung, and prostate cancer is independent of hypertension, hyperlipidemia, and gout in Taiwanese patients. Metabolism 61: 242-249, 2012.

26. Shi Y and Hu FB: The global implications of diabetes and cancer. Lancet 383: 1947-1948, 2014.

27. Yoon JM, Son KY, Eom CS, Durrance D and Park SM: Pre-existing diabetes mellitus increases the risk of gastric cancer: A meta-analysis. World J Gastroenterol 19: 936-945, 2013.

28. Ikeda F, Doi Y, Yonemoto K, Ninomiya T, Kubo M, Shikata K, Hata J, Tanizaki Y, Matsumoto T, Iida M and Kiyohara Y: Hyperglycemia increases risk of gastric cancer posed by Helicobacter pylori infection: A population-based cohort study. Gastroenterology 136: 1234-1241, 2009.

29. Yamagata H, Kiyohara Y, Nakamura S, Kubo M, Tanizaki Y, Matsumoto T, Tanaka K, Kato I, Shirota T and Iida M: Impact of fasting plasma glucose levels on gastric cancer incidence in a general Japanese population: The Hisayama study. Diabetes Care 28: 789-794, 2005

30. Giovannucci E, Harlan DM, Archer MC, Bergenstal RM, Gapstur SM, Habel LA, Pollak M, Regensteiner JG and Yee D: Diabetes and cancer: A consensus report. CA Cancer J Clin 60: 207-221, 2010.

31. Noto H, Goto A, Tsujimoto T, Osame K and Noda M: Latest insights into the risk of cancer in diabetes. J Diabetes Investig 4: 225-232, 2013

32. Stopsack KH, Ziehr DR, Rider JR and Giovannucci EL: Metformin and prostate cancer mortality: A meta-analysis. Cancer Causes Control 27: 105-113, 2016.

33. Belfiore A, Frasca F, Pandini G, Sciacca L and Vigneri R: Insulin receptor isoforms and insulin receptor/insulin-like growth factor receptor hybrids in physiology and disease. Endoc Rev 30: 586-623, 2009.

34. Ikeda F and Kiyohara Y: Helicobacter pylori infection and Hyperglycemia/Diabetes are associated with an increased risk of gastric cancer. Gan To Kagaku Ryoho 42: 529-533, 2015 , (In Japanese).

35. Zhou Y, Wang Y, Wang S and Shen L: Hyperglycemia promotes human gastric carcinoma progression via aquaporin 3. Dig Dis Sci 60: 2338-2345, 2015.

36. Wu L, Zhu J, Prokop LJ and Murad MH: Pharmacologic therapy of diabetes and overall cancer risk and mortality: A meta-analysis of 265 studies. Sci Rep 5: 10147, 2015.

37. Thakkar B, Aronis KN, Vamvini MT, Shields K and Mantzoros CS: Metformin and sulfonylureas in relation to cancer risk in type II diabetes patients: A meta-analysis using primary data of published studies. Metabolism 62: 922-934, 2013.

38. Franciosi M, Lucisano G, Lapice E, Strippoli GF, Pellegrini F and Nicolucci A: Metformin therapy and risk of cancer in patients with type 2 diabetes: Systematic review. PLoS One 8: e71583, 2013.

39. Soranna D, Scotti L, Zambon A, Bosetti C, Grassi G, Catapano A, La Vecchia C, Mancia G and Corrao G: Cancer risk associated with use of metformin and sulfonylurea in type 2 diabetes: A meta-analysis. Oncologist 17: 813-822, 2012.

40. Noto H, Goto A, Tsujimoto T and Noda M: Cancer risk in diabetic patients treated with metformin: A systematic review and meta-analysis. PLoS One 7: e33411, 2012.

41. Sciarretta S, Volpe M and Sadoshima J: Mammalian target of rapamycin signaling in cardiac physiology and disease. Circ Res 114: 549-564, 2014

42. Pasello G, Urso L, Conte P and Favaretto A: Effects of sulfonylureas on tumor growth: A review of the literature. Oncologist 18: $1118-1125,2013$.

43. Yu G, Fang W, Xia T, Chen Y, Gao Y, Jiao X, Huang S, Wang J, $\mathrm{Li} \mathrm{Z}$ and Xie K: Metformin potentiates rapamycin and cisplatin in gastric cancer in mice. Oncotarget 6: 12748-12762, 2015. 
44. Dowling RJ, Niraula S, Chang MC, Done SJ, Ennis M McCready DR, Leong WL, Escallon JM, Reedijk M, Goodwin PJ and Stambolic V: Changes in insulin receptor signaling underlie neoadjuvant metformin administration in breast cancer: A prospective window of opportunity neoadjuvant study. Breast Cancer Res 17: 32, 2015.

45. Crino PB, Nathanson KL and Henske EP: The tuberous sclerosis complex. N Engl J Med 355: 1345-1356, 2006.

46. Huang $\mathrm{J}$ and Manning BD: A complex interplay between Akt, TSC2 and the two mTOR complexes. Biochem Soc Trans 37 217-222, 2009.

47. Qin L, Wang Z, Tao L and Wang Y: ER stress negatively regulates AKT/TSC/mTOR pathway to enhance autophagy. Autophagy 6: 239-247, 2014

48. Zhou JY, Xu B and Li L: A new role for an old drug: Metformin targets MicroRNAs in treating diabetes and cancer. Drug Dev Res 76: 263-269, 2015.

49. Pollak M: Insulin and insulin-like growth factor signalling in neoplasia. Nat Rev Cancer 8: 915-928, 2008.

50. Monami M, Lamanna C, Balzi D, Marchionni N and Mannucci E: Sulphonylureas and cancer: A case-control study. Acta Diabetol 46: 279-284, 2009.

51. Núñez M, Medina V, Cricco G, Croci M, Cocca C, Rivera E, Bergoc R and Martín G: Glibenclamide inhibits cell growth by inducing G0/G1 arrest in the human breast cancer cell line MDA-MB-231. BMC Pharmacol Toxicol 14: 6, 2013.

52. Zhanping W, Xiaoyu P, Na C, Shenglan W and Bo W: Voltage-gated $\mathrm{K}+$ channels are associated with cell proliferation and cell cycle of ovarian cancer cell. Gynecol Oncol 104 455-460, 2007.

53. Abdul M and Hoosein N: Voltage-gated potassium ion channels in colon cancer. Oncol Rep 9: 961-964, 2002.
54. Malhi H, Irani AN, Rajvanshi P, Suadicani SO, Spray DC, McDonald TV and Gupta S: KATP channels regulate mitogenically induced proliferation in primary rat hepatocytes and human liver cell lines. Implications for liver growth contro and potential therapeutic targeting. J Biol Chem 275: 26050-26057, 2000.

55. Bonnet S, Archer SL, Allalunis-Turner J, Haromy A, Beaulieu C, Thompson R, Lee CT, Lopaschuk GD, Puttagunta L, Bonnet S, et al: A mitochondria-K+ channel axis is suppressed in cancer and its normalization promotes apoptosis and inhibits cancer growth. Cancer Cell 11: 37-51, 2007.

56. Felipe A, Vicente R, Villalonga N, Roura-Ferrer M, Martínez-Mármol R, Solé L, Ferreres JC and Condom E: Potassium channels: New targets in cancer therapy. Cancer Detect Prev 30: 375-385, 2006.

57. Conti M: Targeting K+ channels for cancer therapy. J Exp Ther Oncol 4: 161-166, 2004.

58. Ardehali $\mathrm{H}$ and $\mathrm{O}^{\prime}$ Rourke B: Mitochondrial K(ATP) channels in cell survival and death. J Mol Cell Cardiol 39: 7-16, 2005.

59. Yang X, So WY, Ma RC, Yu LW, Ko GT, Kong AP, Ng VW, Luk AO, Ozaki R, Tong PC, et al: Use of sulphonylurea and cancer in type 2 diabetes-The Hong Kong Diabetes Registry. Diabetes Res Clin Pract 90: 343-351, 2010.

60. Elwood PC, Gallagher AM, Duthie GG, Mur LA and Morgan G: Aspirin, salicylates, and cancer. Lancet 373: 1301-1309, 2009.

61. Jamshidinaeini Y, Akbari ME, Abdollahi M, Ajami M and Davoodi SH: Vitamin D status and risk of breast cancer in Iranian women: A case-control study. J Am Coll Nutr 35: 639-646, 2016.

62. Osmak M: Statins and cancer: Current and future prospects. Cancer Lett 324: 1-12, 2012. 